\title{
An Inhibitor-Tethered Resin for Detection of Active Matrix Metalloproteinases Involved in Disease
}

Dusan Hesek, Marta Toth, Viktor Krchnak, Rafael Fridman and Shahriar Mobashery*

Department of Chemistry and Biochemistry and the Walther Cancer Research Center, University of Notre Dame, Notre Dame, IN 45665 and

Department of Pathology, Wayne State University School of Medicine,

Detroit, MI 48201

mobashery@nd.edu

\section{Supporting Information}

Table of Contents.

Fig $1:{ }^{1} \mathrm{H}$ NMR of $7 \ldots \ldots \ldots \ldots \ldots \ldots \ldots \ldots \ldots \ldots \ldots \ldots \ldots \ldots \ldots \ldots \ldots \ldots$, S2

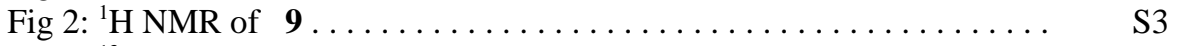

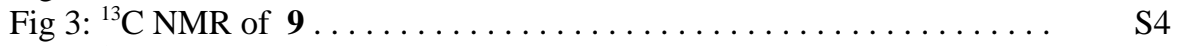

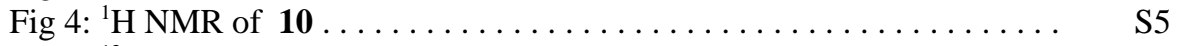

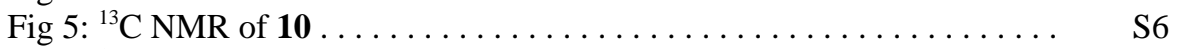

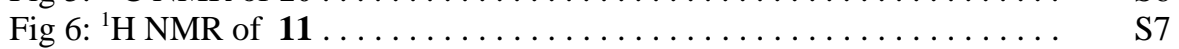

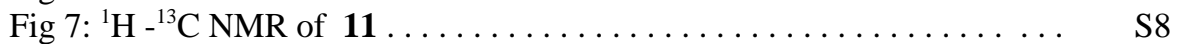

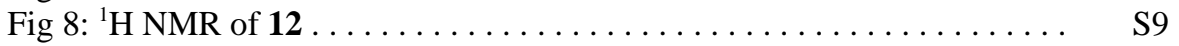

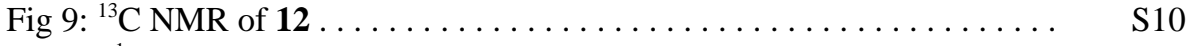

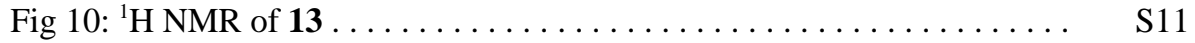

Fig $11:{ }^{13} \mathrm{C}$ NMR of $13 \ldots \ldots \ldots \ldots \ldots \ldots \ldots \ldots \ldots \ldots \ldots \ldots \ldots \ldots \ldots \ldots \ldots \ldots \ldots, \quad$ S12

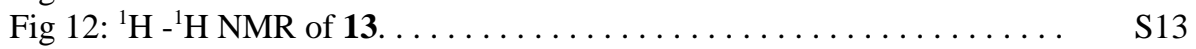

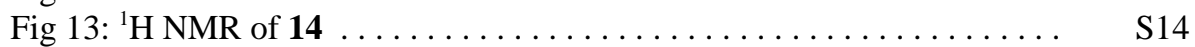

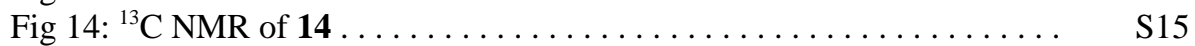

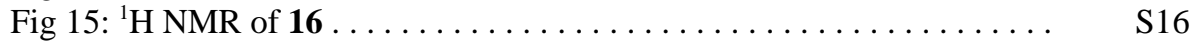

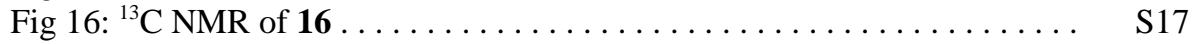

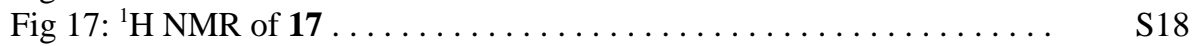

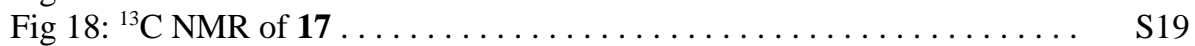

HRMS (FAB) of $7, \mathbf{9}, \mathbf{1 0}, \mathbf{1 1}, \mathbf{1 2}, \mathbf{1 3}, \mathbf{1 4}, \mathbf{1 6}$ and $17 \ldots \ldots \ldots \ldots \ldots \ldots$ S20-S24 
Figure 1: $1 \mathrm{H}$ NMR of 7

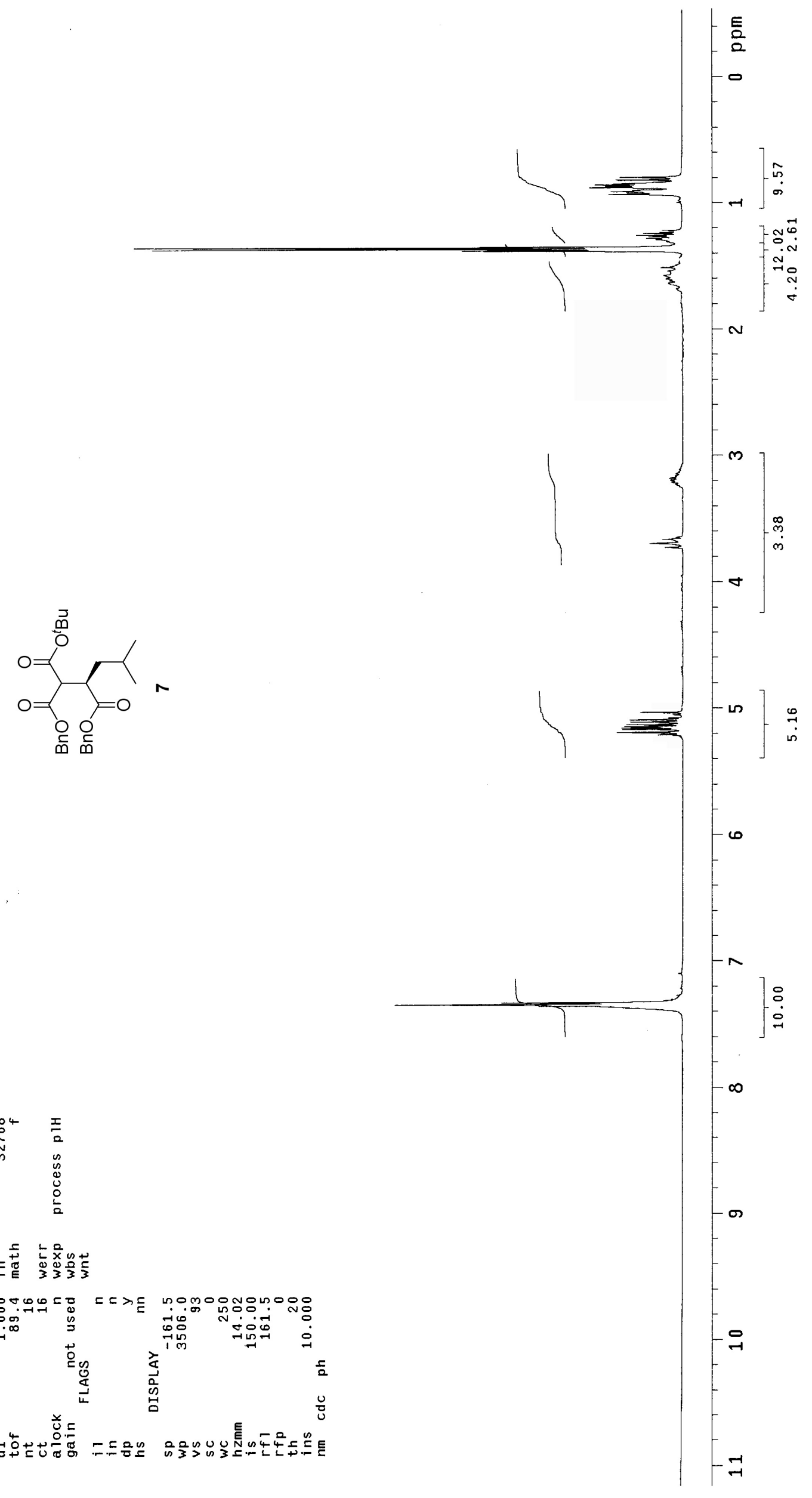


Figure 2: $1 \mathrm{H}$ NMR of 9

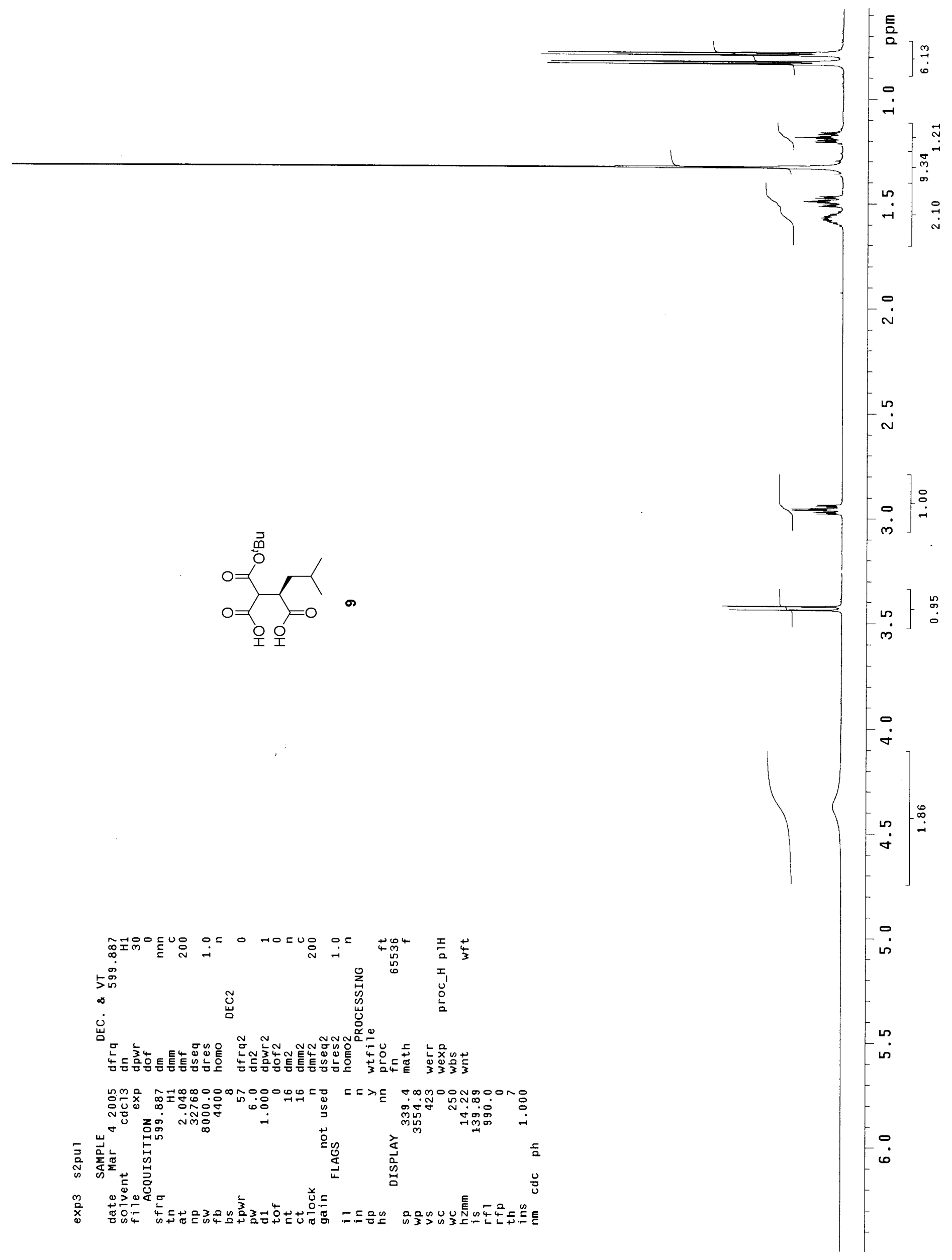


Figure 3: $13 \mathrm{C}$ NMR of 9

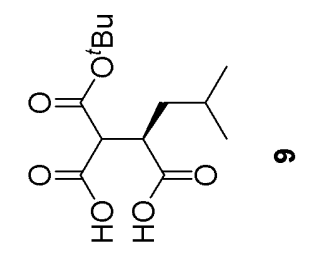

$s \varepsilon \mathcal{E} \cdot\llcorner z-$

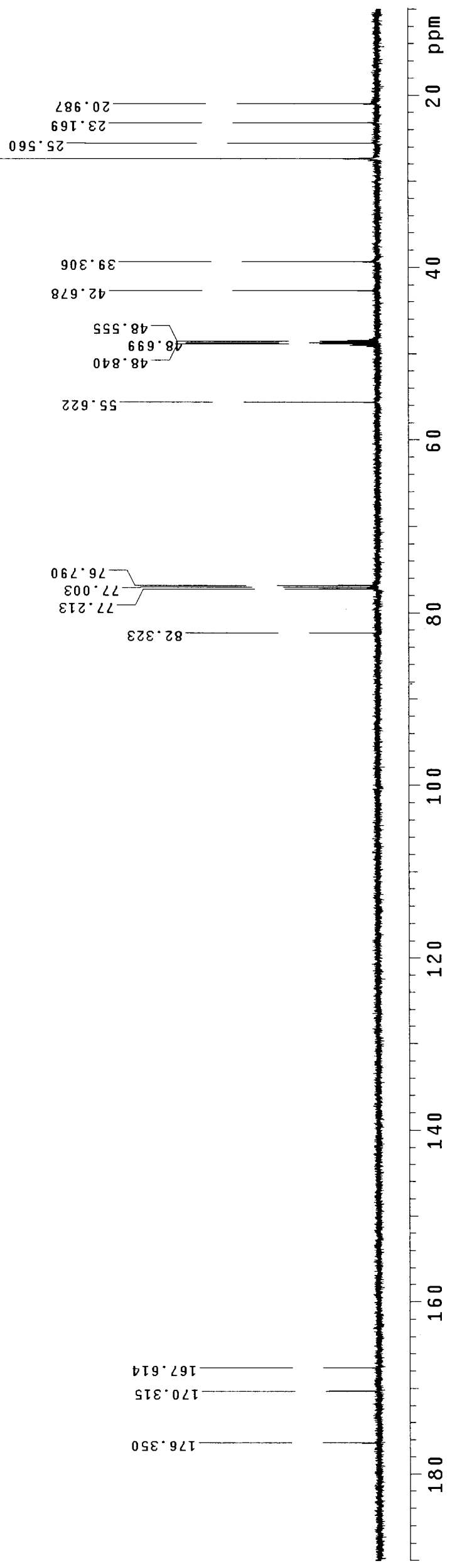


Figure 4: $1 \mathrm{H}$ NMR of 10

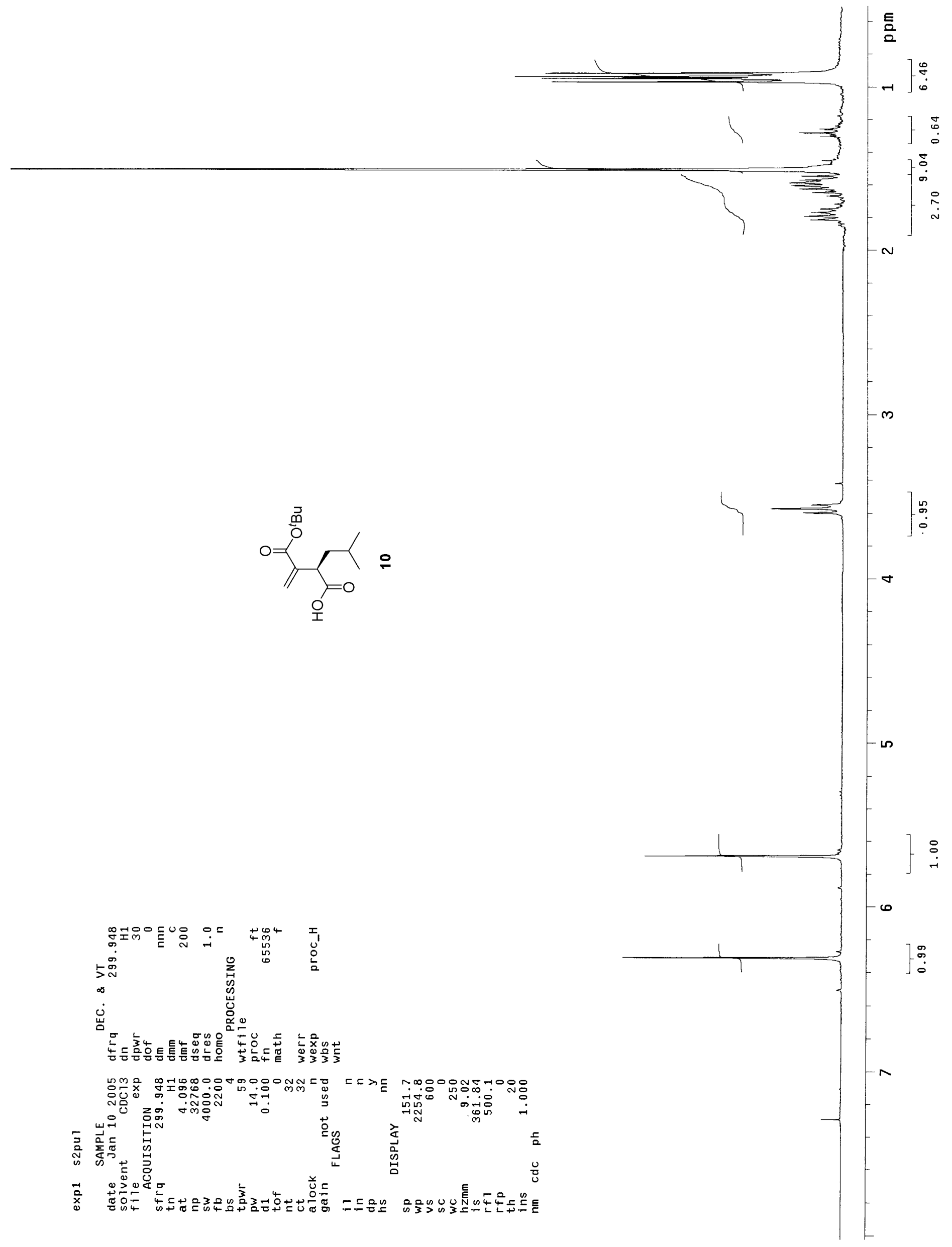


Figure 5: 13C NMR of 10

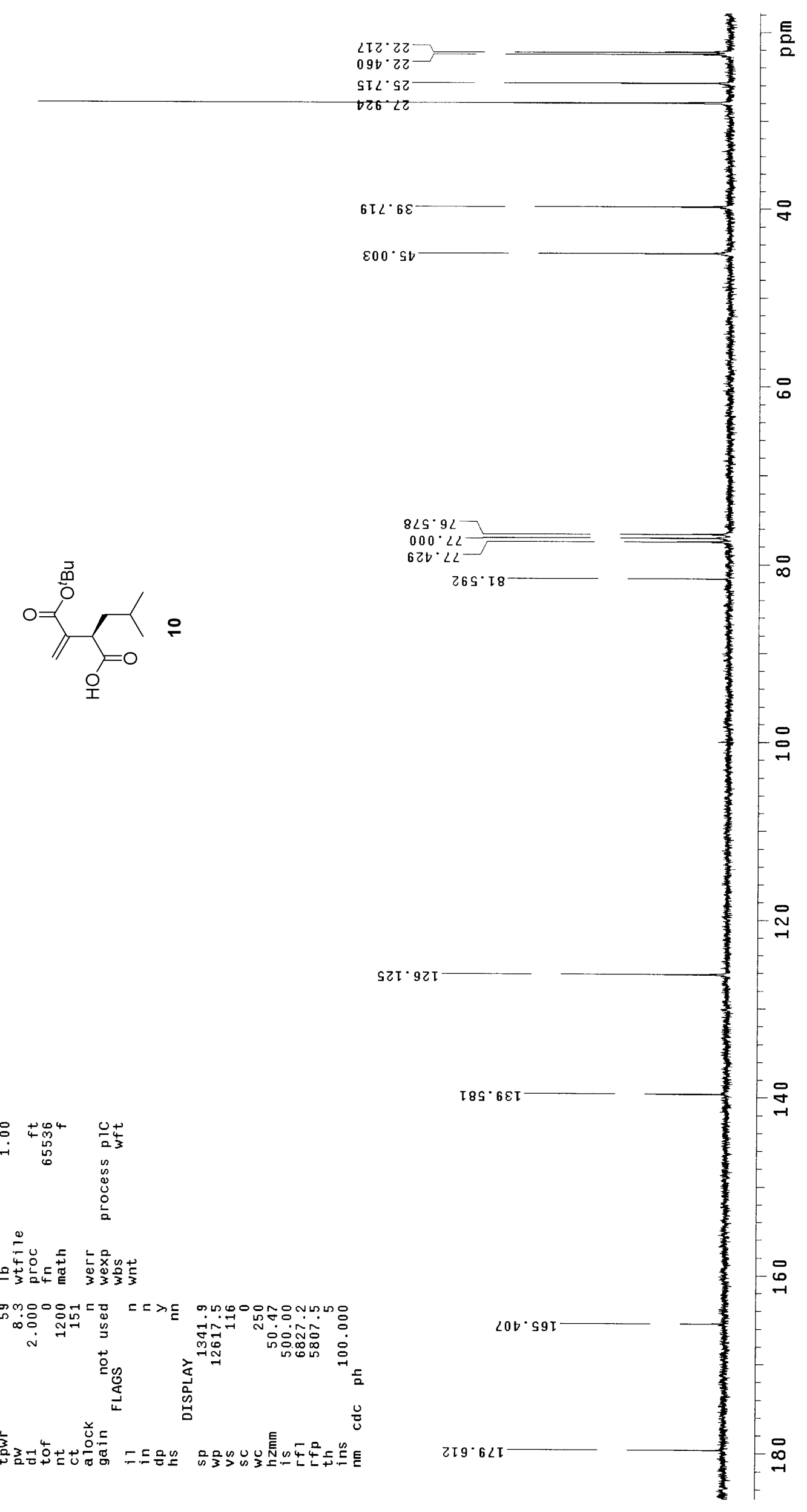


Figure 6: 1H NMR of 11

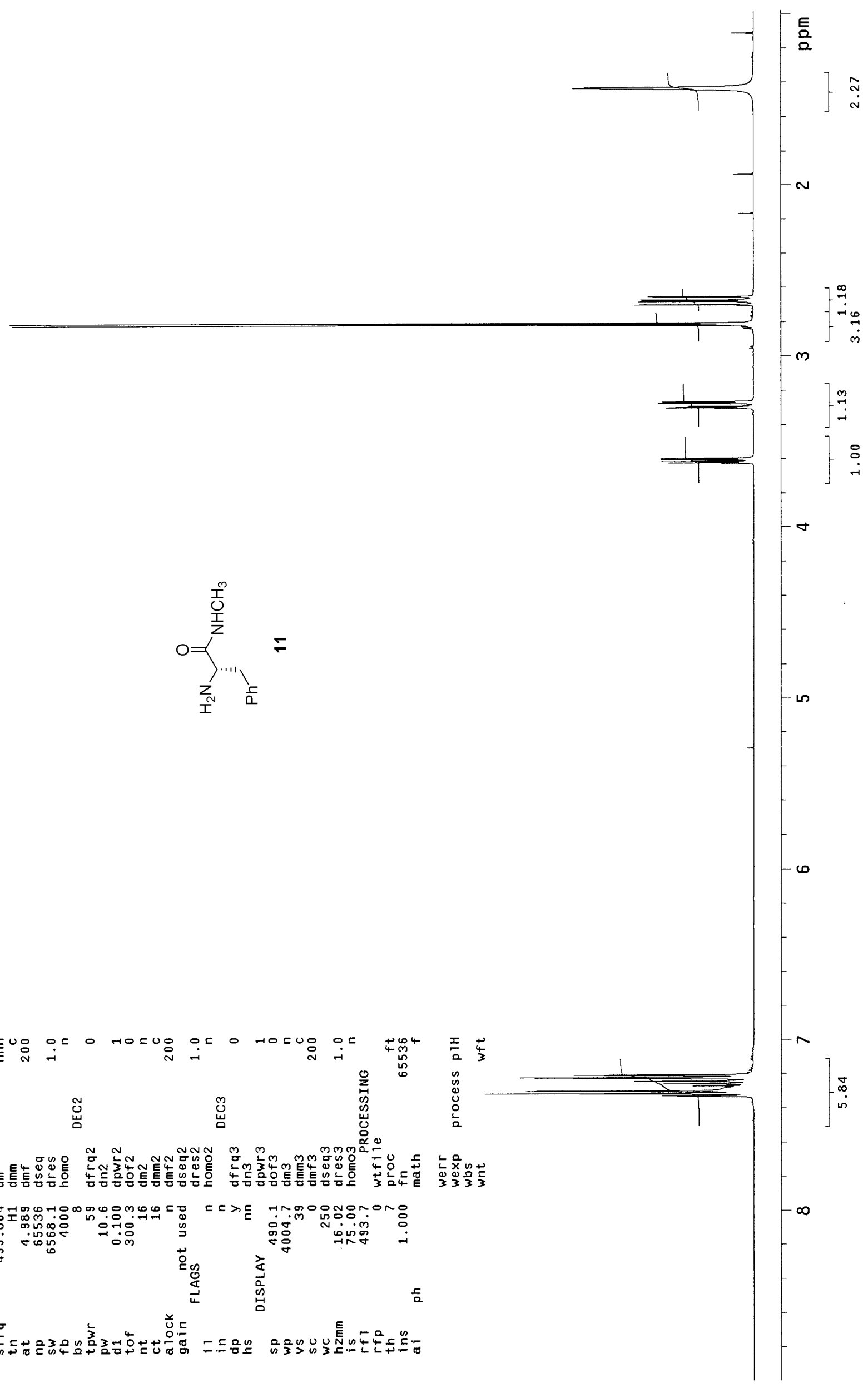


Figure 7: $\mathrm{H}-\mathrm{C}$ (hetcor) NMR of 11

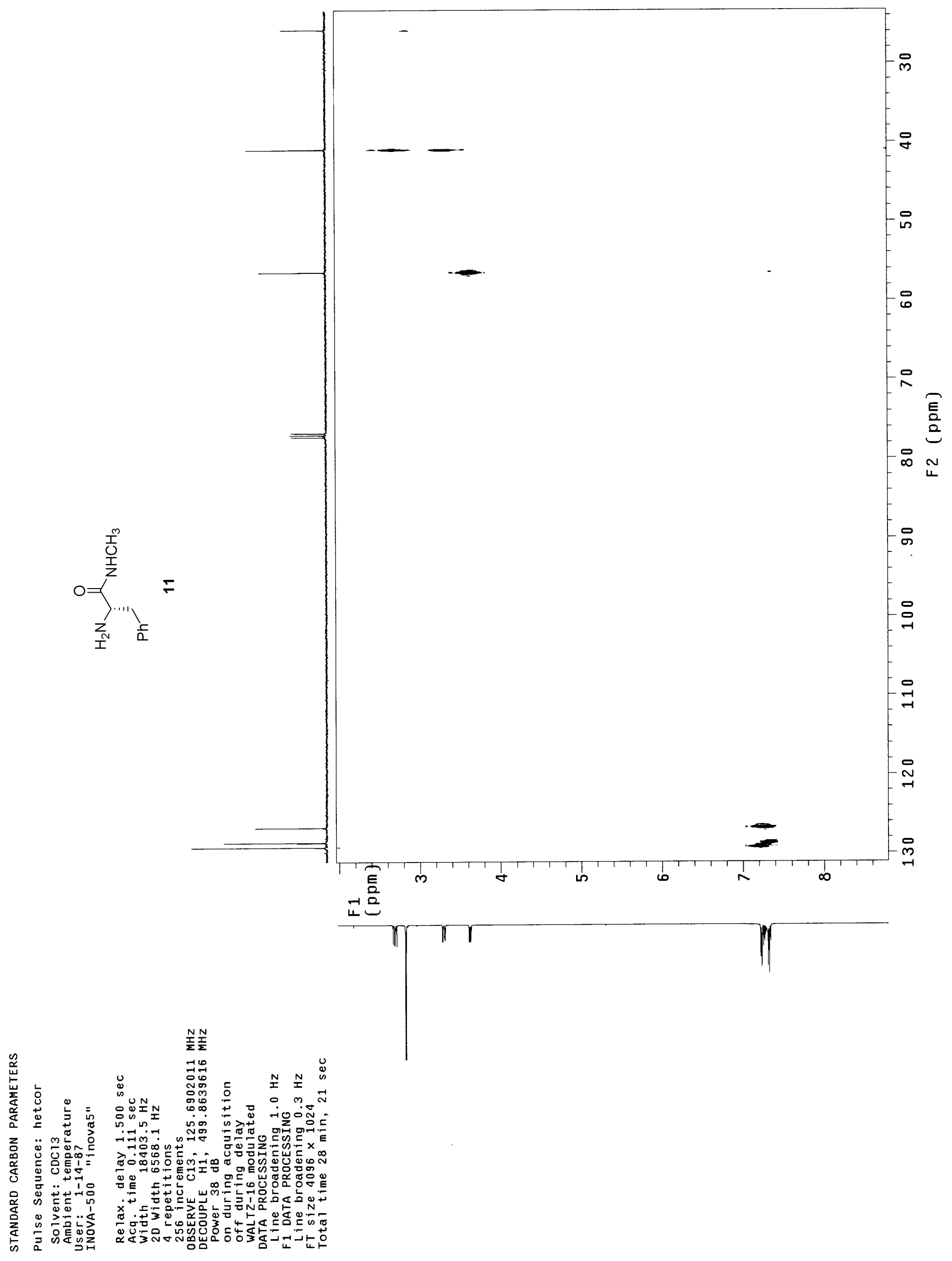


Figure 8: $1 \mathrm{H}$ NMR of 12
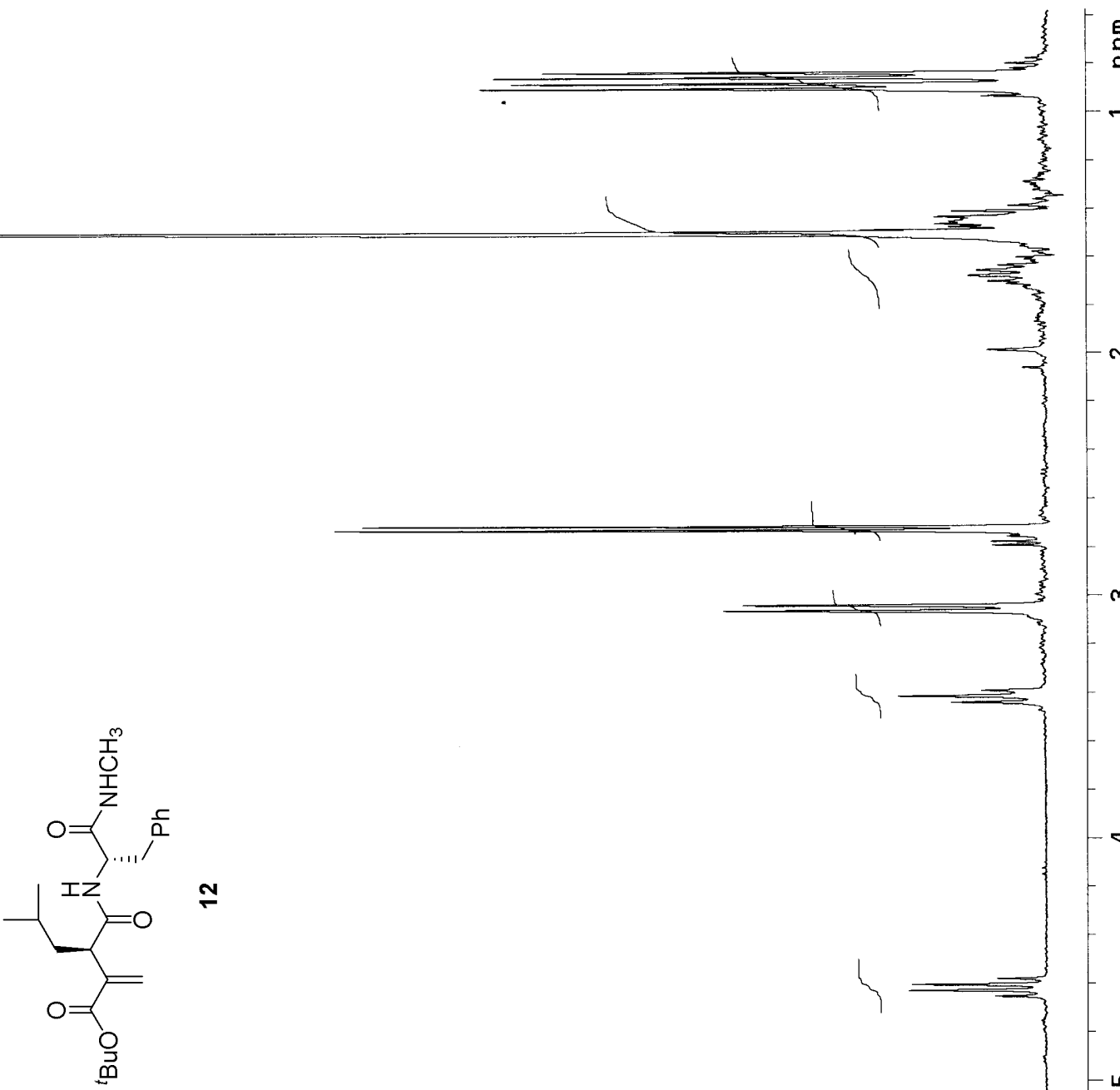

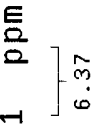

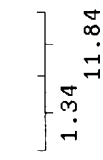

$N$

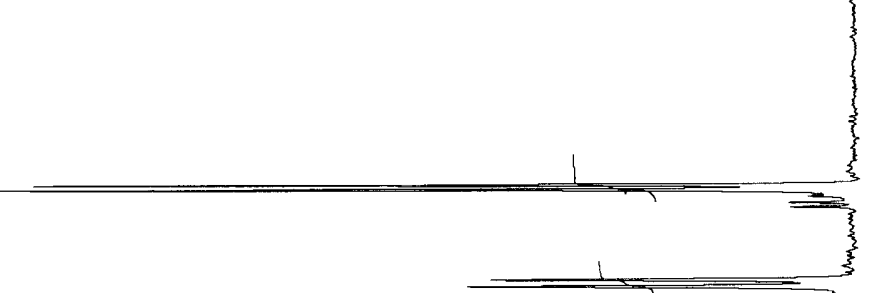

$f \stackrel{\tilde{\omega}}{\dot{\alpha}}$

$m] \stackrel{\tilde{N}}{0}$

]$\stackrel{\infty}{\stackrel{-}{-}}$

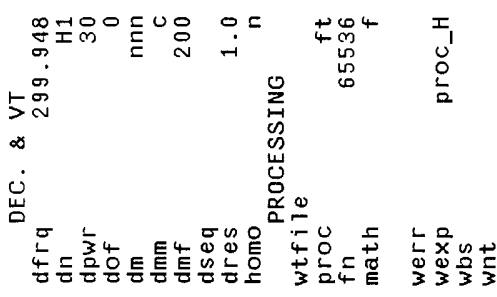

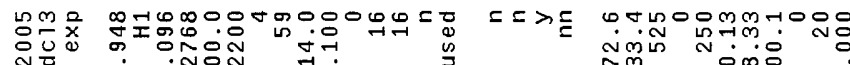

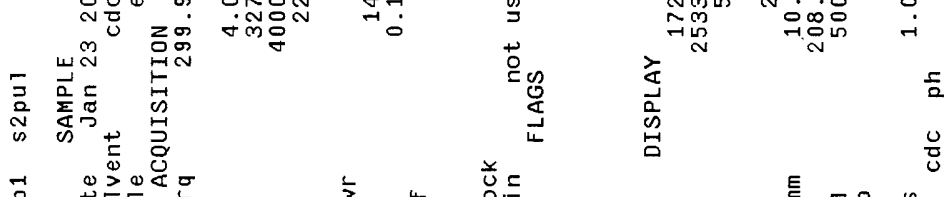

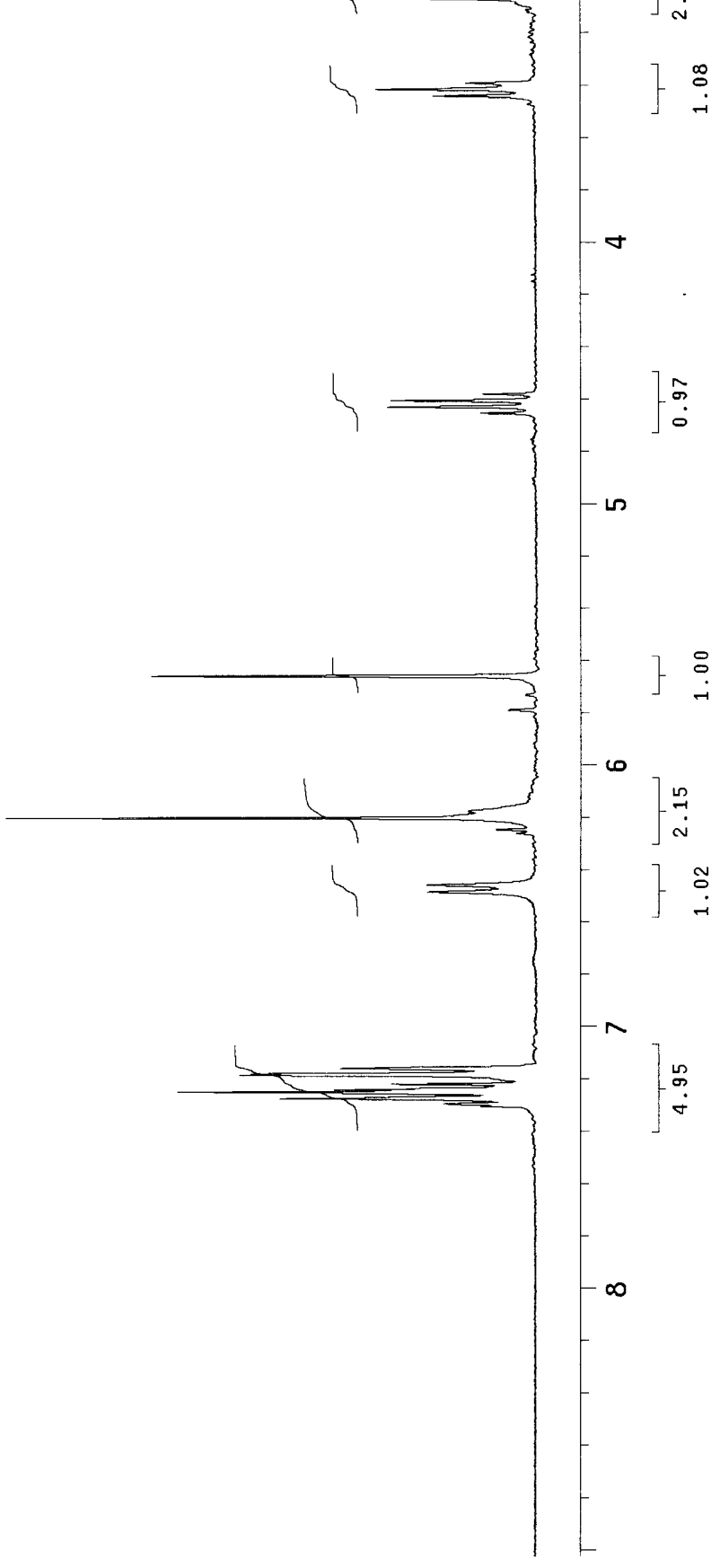


Figure 9: $13 \mathrm{C}$ NMR of 12
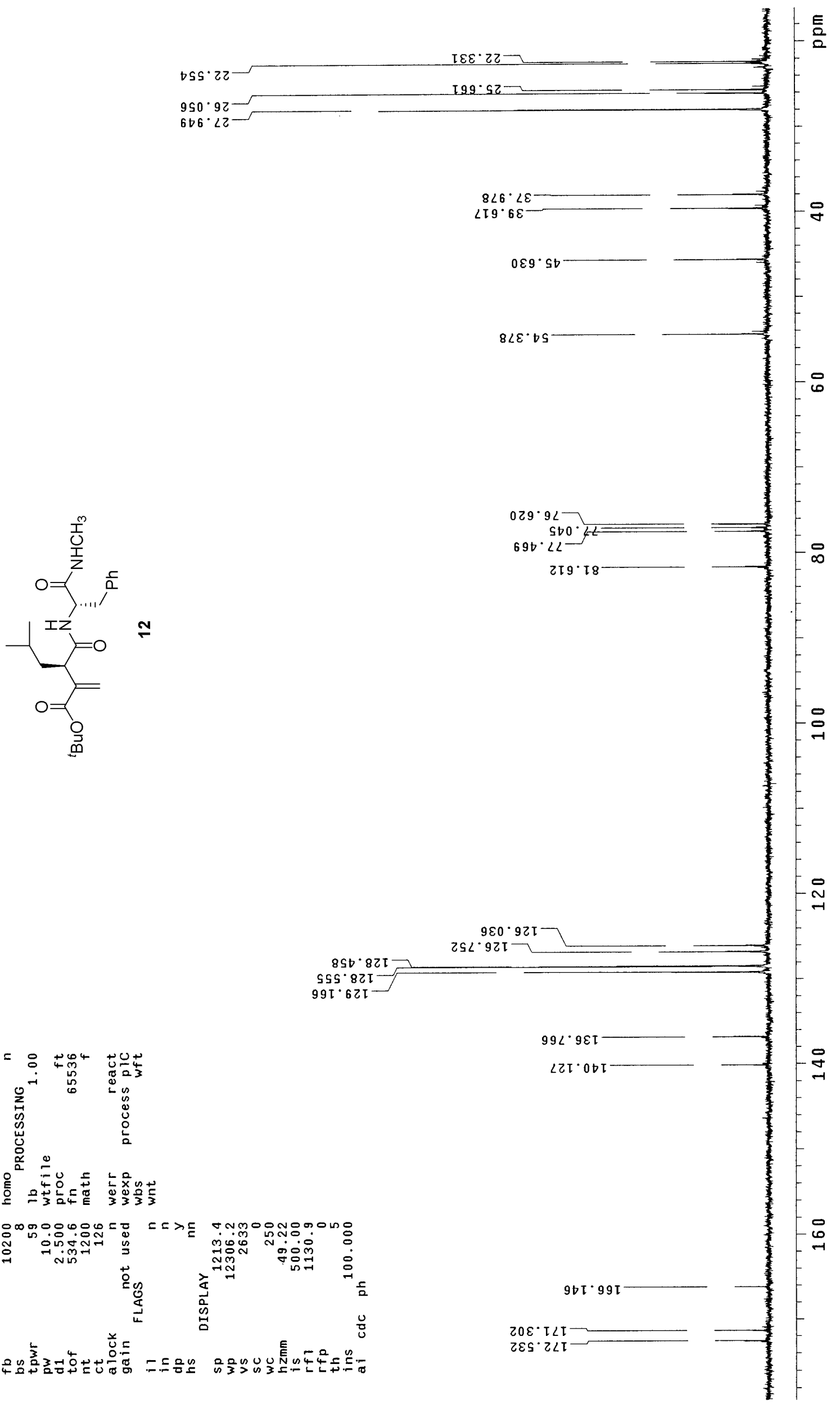
Figure 10: $1 \mathrm{H}$ NMR of 13

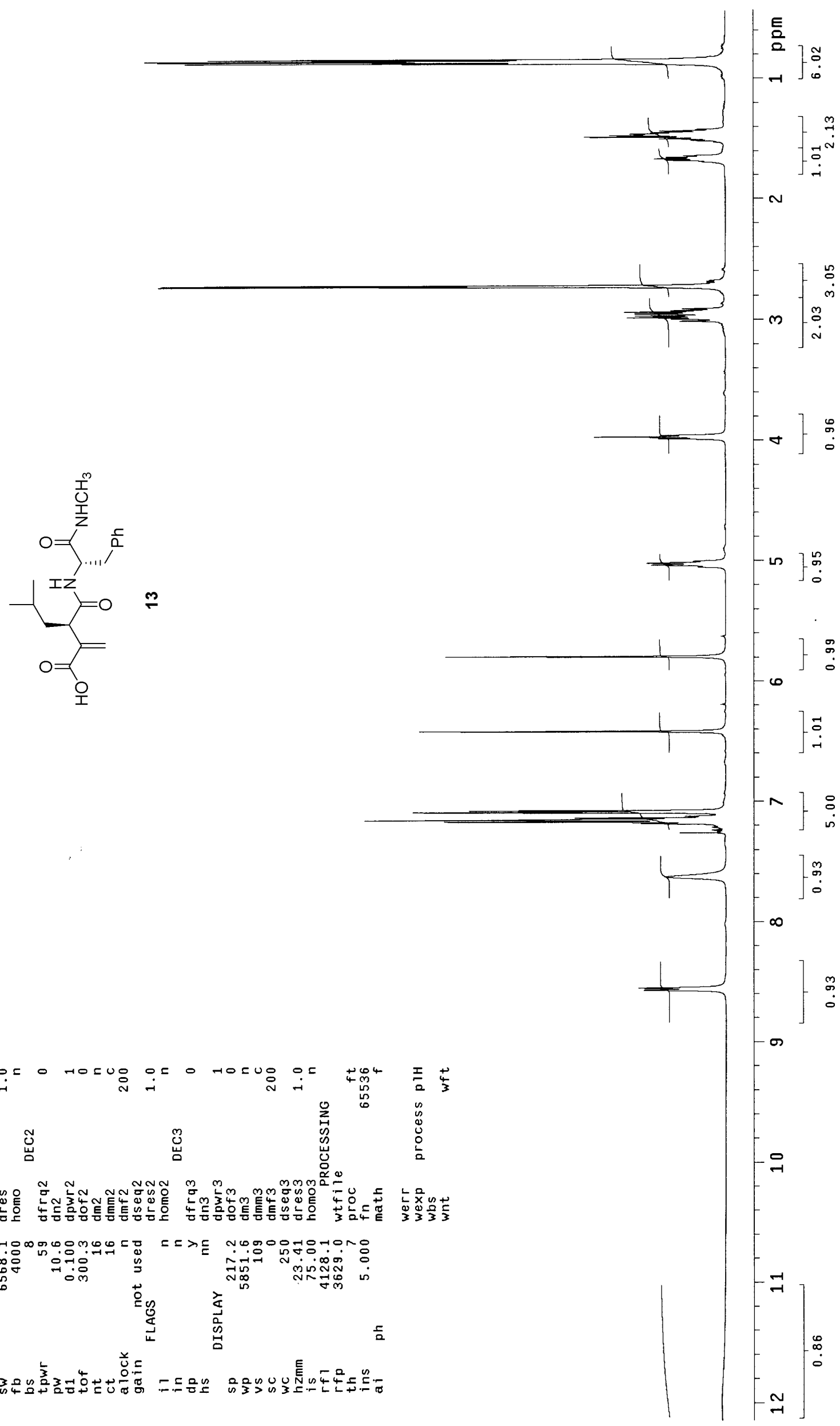


Figure 11: 13C NMR of 13

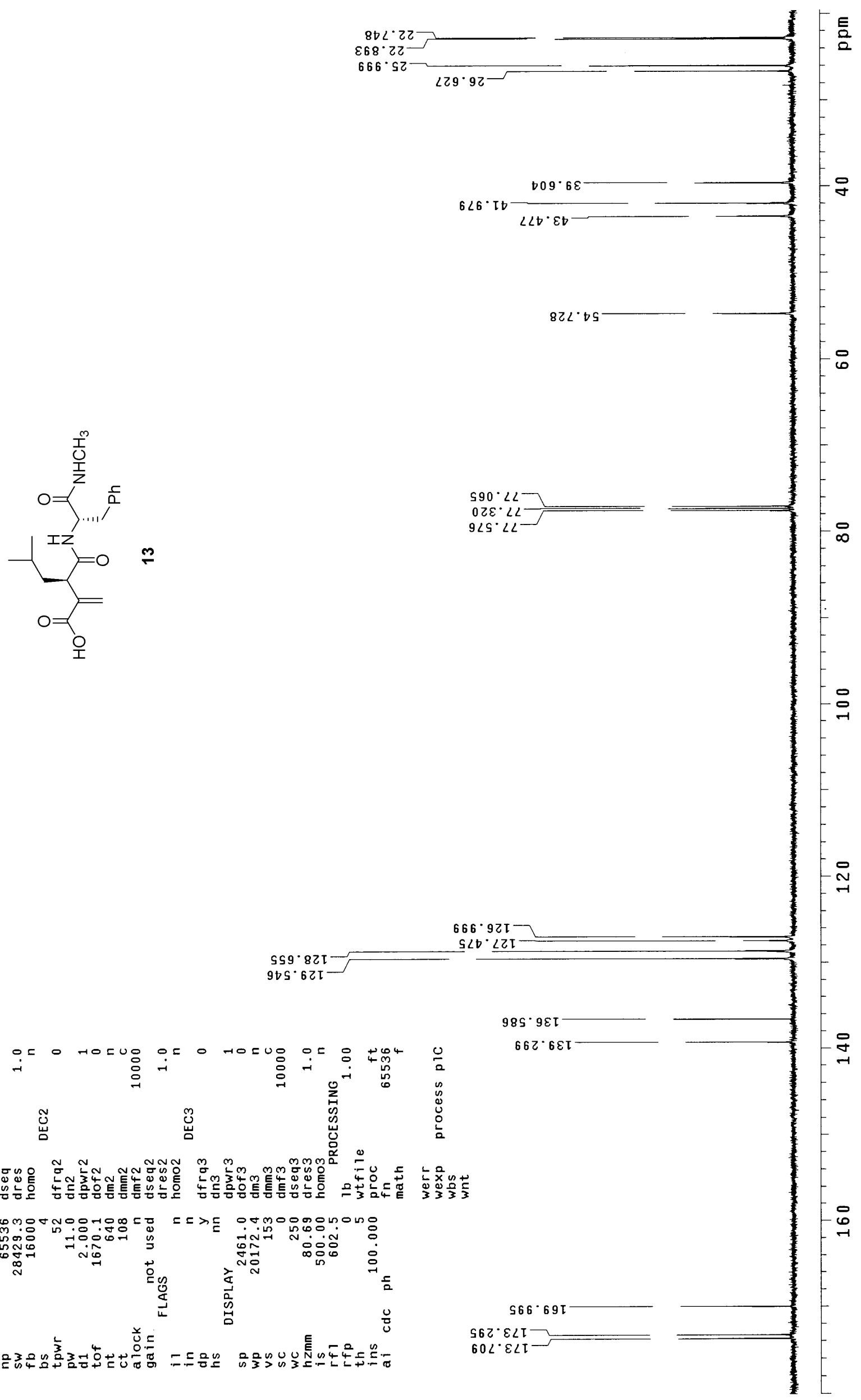


Figure 12: $\mathrm{H}-\mathrm{H}$ cosy NMR of 13

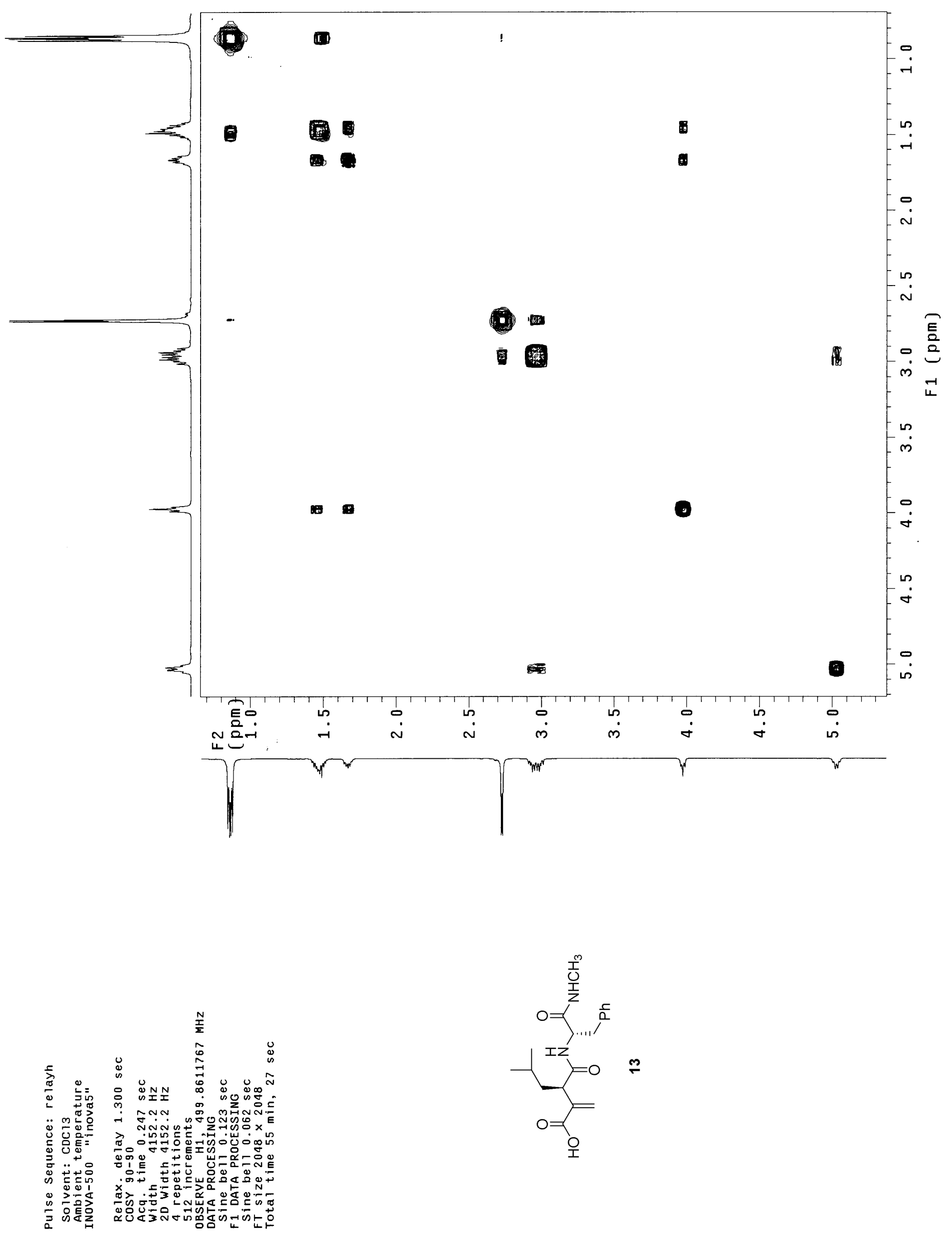


Figure 13: $1 \mathrm{H}$ NMR of 14

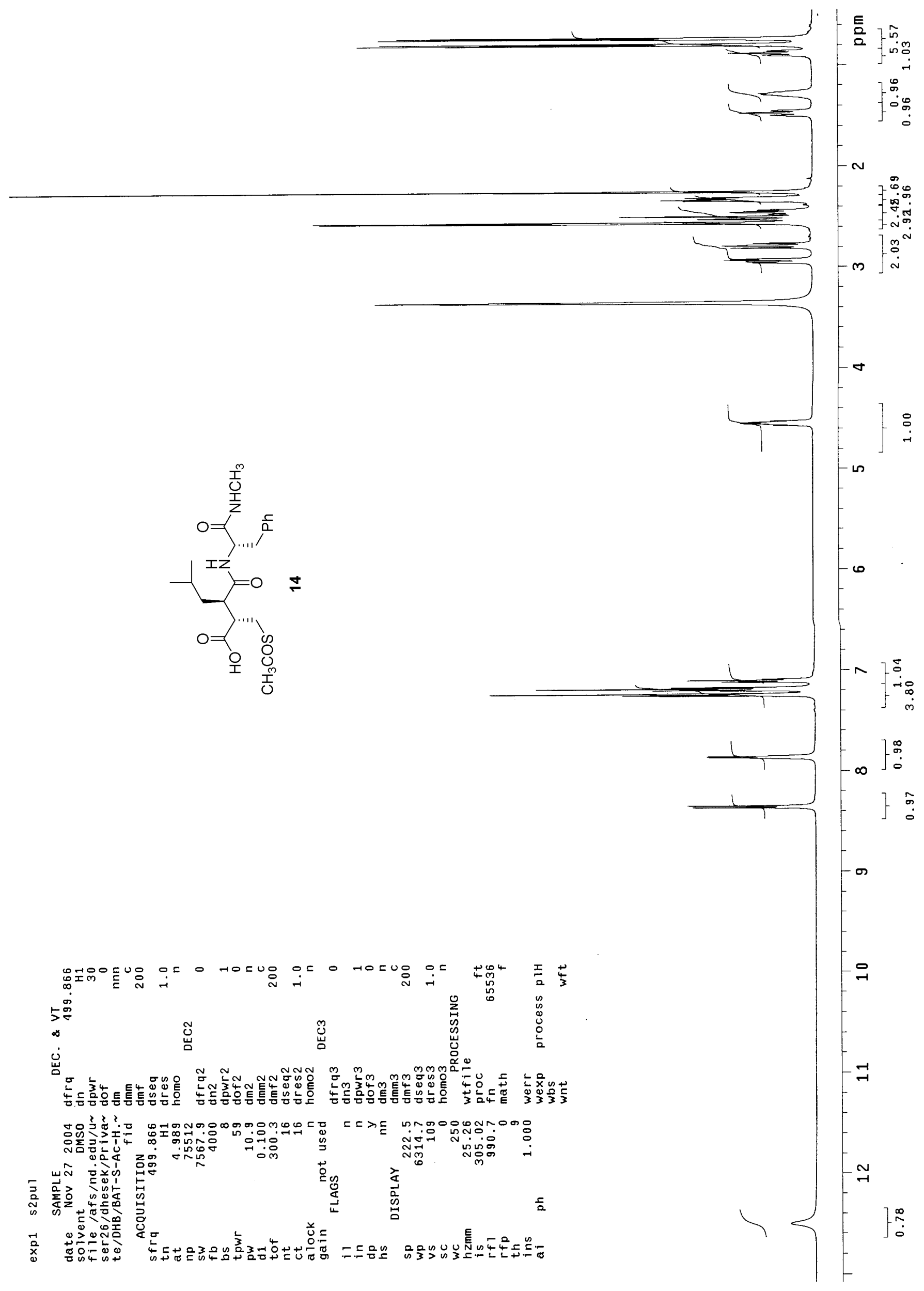


Figure 14: $13 \mathrm{C}$ NMR of 14

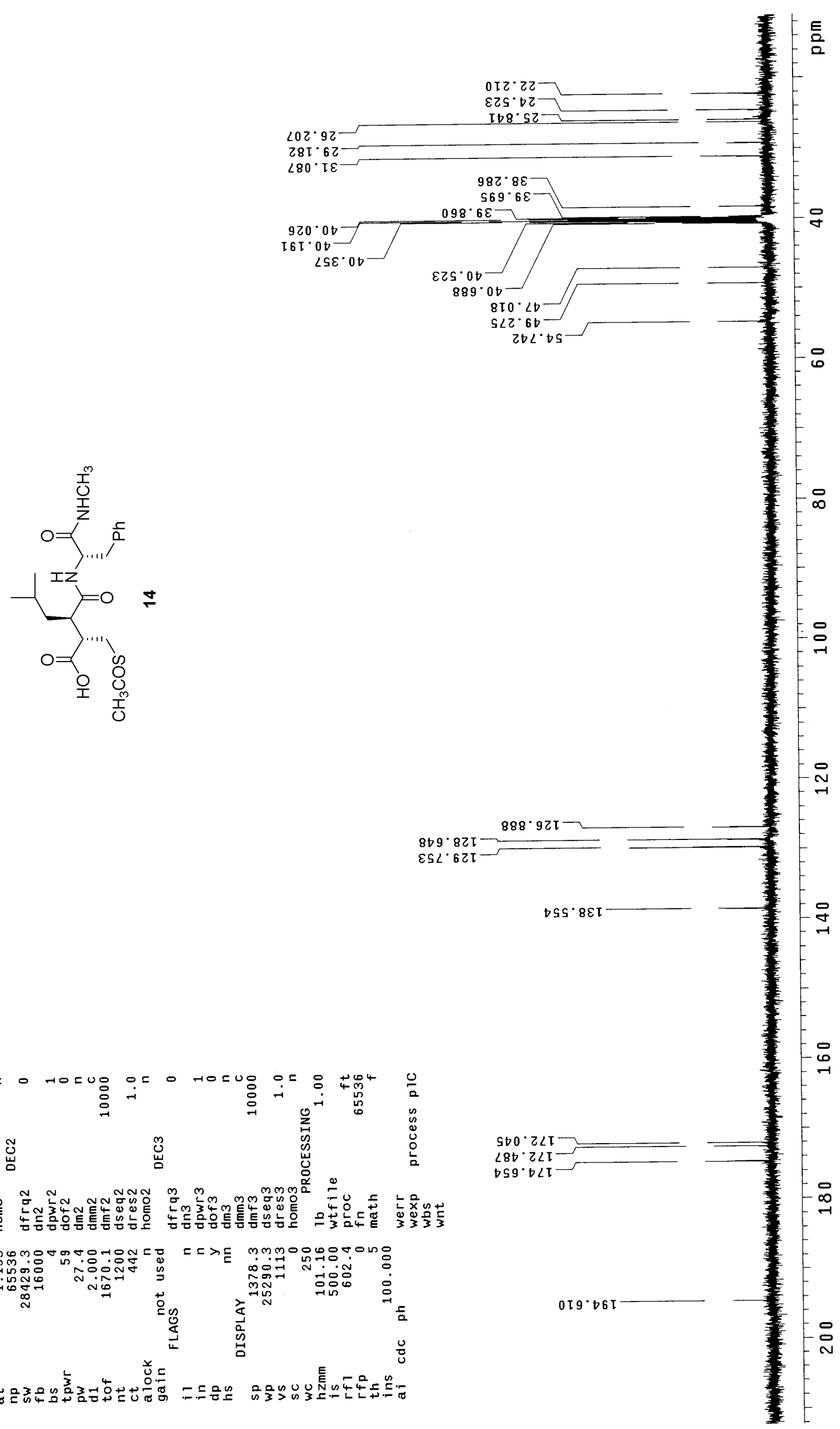


Figure 15: $1 \mathrm{H}$ NMR of 16

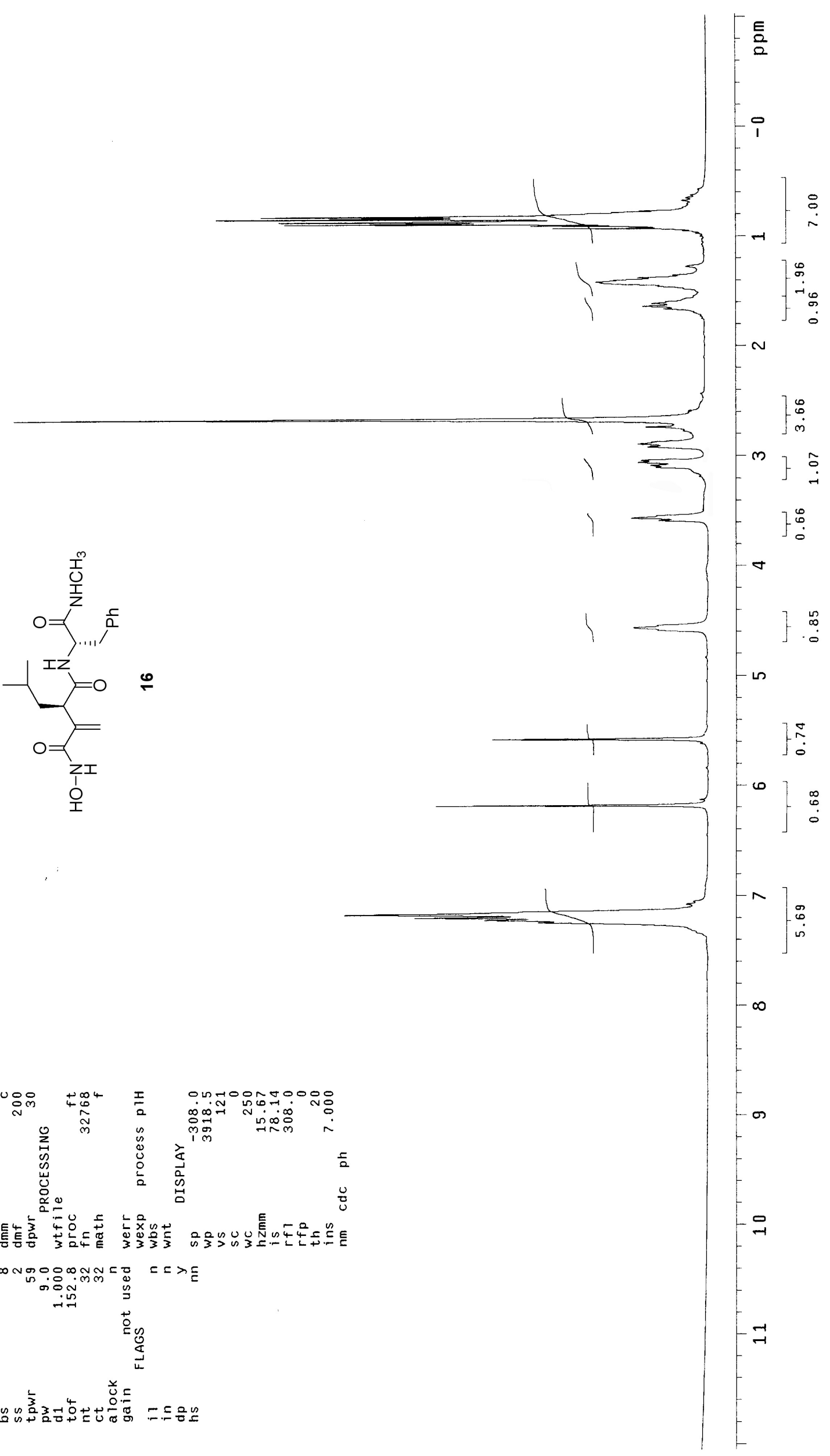


Figure 16: $13 \mathrm{C}$ NMR of 16

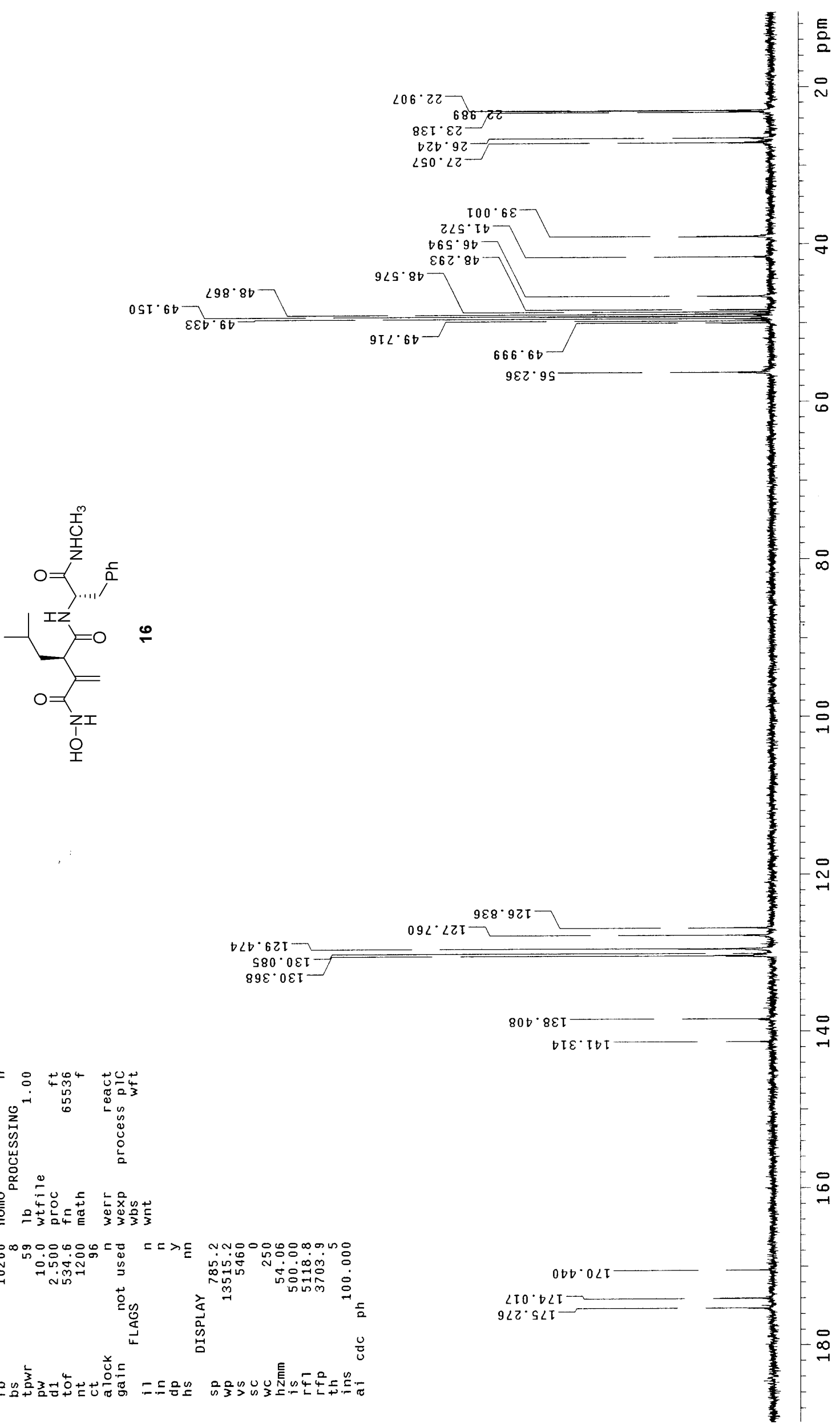


Figure 17: $1 \mathrm{H}$ NMR of 17

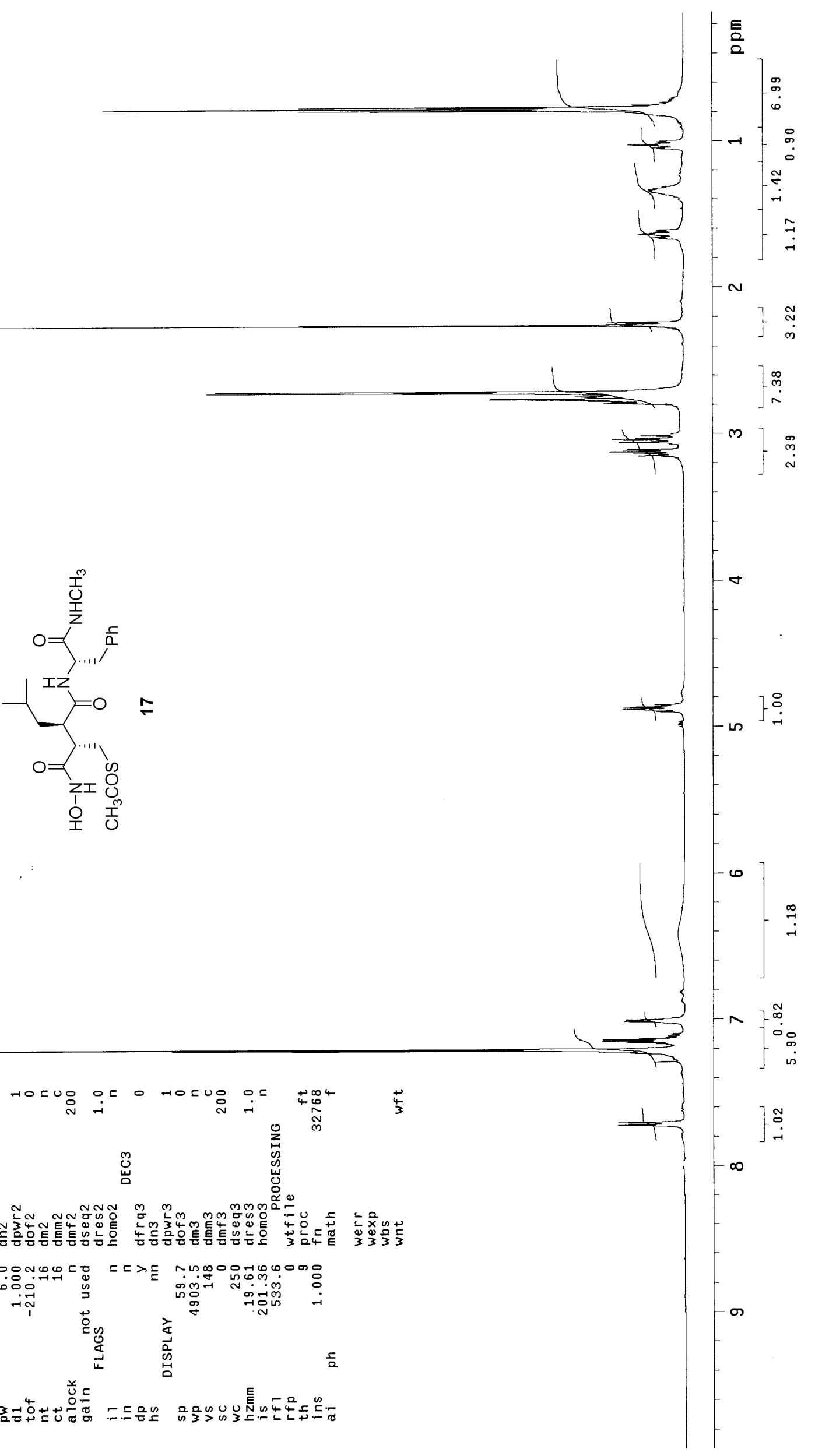


Figure 18: $13 \mathrm{C}$ NMR of 17

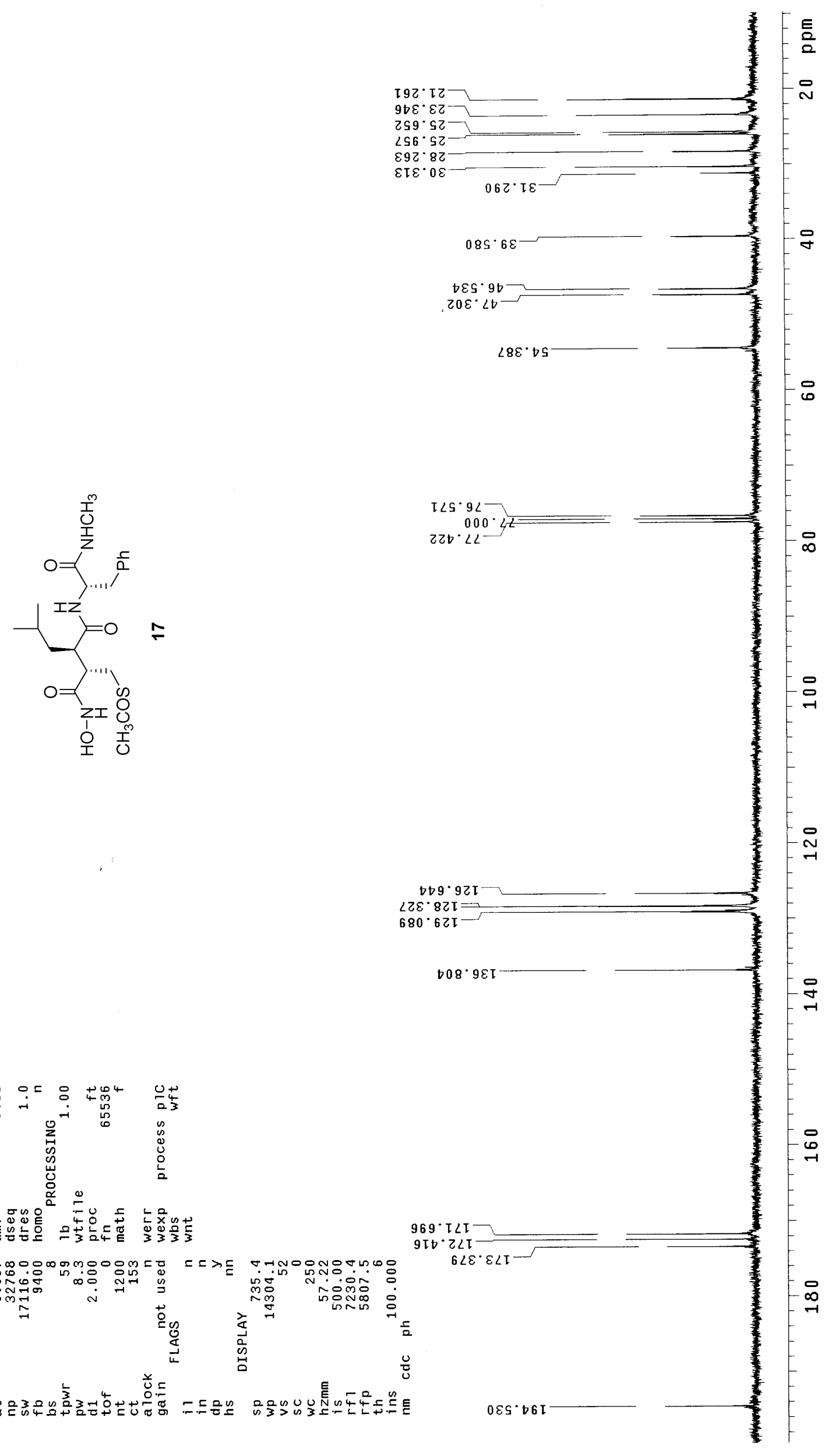




\section{HRMS for 7}

[ Elemental Composition ]

Data : J6.621hr-001

Date : 21-Mar-2006 14:11

Page: 1

Sample: DHR-5

Note : - in PEG standard

Inlet : Reserv.

$\mathrm{RT}: 0.27 \mathrm{~min}$

Elements : C $60 / 0, \mathrm{H} 90 / 0, \mathrm{O} 6 / 0$

Mass Tolerance : $3 \mathrm{mmu}$

Unsaturation (U.S.) : $-0.5-12.0$

Observed $\mathrm{m} / \mathrm{z}$ Int\% Err [ppm / mmu ] U.S. Composition $\begin{array}{llllllll}455.2443 & 100.0 & +2.0 / & +0.9 & 10.5 & \text { C } 27 \text { H } 3506\end{array}$

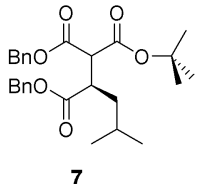

Chemical Formula: $\mathrm{C}_{27} \mathrm{H}_{34} \mathrm{O}_{6}$ Exact Mass: 454.24

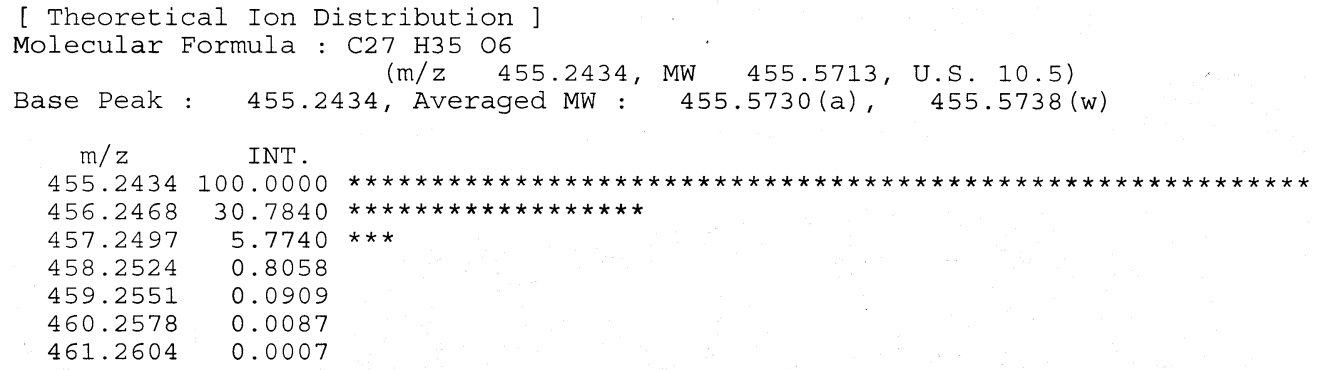

\section{HRMS for 9}

[ Elemental Composition ]

Data : J6.622hr-001

Sample: DHR-3

Note : - in PEG standard

Inlet : Reserv.

$\mathrm{RT}: 0.27 \mathrm{~min}$

Elements : C $60 / 0, \mathrm{H} 90 / 0, \mathrm{O} 6 / 0$

Mass Tolerance : $3 \mathrm{mmu}$

Unsaturation (U.S.) : $-0.5-12.0$

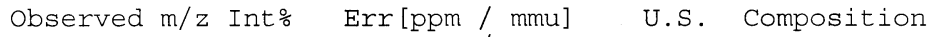
$\begin{array}{lllllll}275.1479 & 6.0 & -5.6 / & -1.5 & 2.5 & \text { C } 13 \mathrm{H} 2306\end{array}$

Page: 1

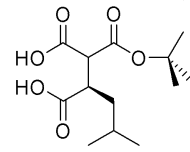

9

Chemical Formula: $\mathrm{C}_{13} \mathrm{H}_{22} \mathrm{O}_{6}$ Exact Mass: 274.14

[ Theoretical Ion Distribution ]

Molecular Formula : C13 H23 O6

$(\mathrm{m} / \mathrm{z} \quad 275.1495, \mathrm{MW} \quad 275.3220$, U.S. 2.5)

Base Peak : 275.1495, Averaged MW : 275.3228(a), 275.3235(w)

$\mathrm{m} / \mathrm{z} \quad \mathrm{INT}$.

275.1495100 .0000

$276.1529 \quad 15.0326$

277.15492 .2523 *

$278.1577 \quad 0.2255$

$279.1600 \quad 0.0199$

$280.1625 \quad 0.0015$ 


\section{HRMS for 10}

[ Elemental Composition ]

Data : J6.619hr-001

Sample: DHR-9

Note : - in PEG standard

Inlet : Reserv.

$\mathrm{RT}: 0.27 \mathrm{~min}$

Elements : C $60 / 0, \mathrm{H} 90 / 0, \mathrm{O} 6 / 0$

Ion Mode : FAB+

Mass Tolerance : $3 \mathrm{mmu}$

Unsaturation (U.S.) : $-0.5-10.0$

Date : 21-Mar-2006 14:05

Page: 1

Observed $\mathrm{m} / \mathrm{z}$ Int $\%$ Err [ppm / mmu] U.S. Composition $\begin{array}{llllll}243.1606+19.0+4.1 / 4+1.0 & 2.5 & \text { C } 13 \mathrm{H} 2304\end{array}$

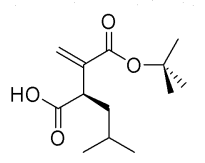

10

Chemical Formula: $\mathrm{C}_{13} \mathrm{H}_{22} \mathrm{O}_{4}$ Exact Mass: 242.15

[ Theoretical Ion Distribution ]

Page: 1

Molecular Formula : C13 H23 O4

$(\mathrm{m} / \mathrm{z} 243.1596, \mathrm{MW} 243.3232, \mathrm{U} . \mathrm{S} .2 .5)$

Base Peak : 243.1596, Averaged MW : $243.3241(\mathrm{a}), \quad 243.3249(\mathrm{w})$

$\mathrm{m} / \mathrm{z}$ INT.

243.1596100 .0000

$244.1631 \quad 14.9565$

245.1654 1.8399*

$246.1680 \quad 0.1640$

$247.1705 \quad 0.0120$

$248.1730 \quad 0.0007$

\section{HRMS for 11}

[ Elemental Composition ]

Data : J6.618hr-001

Date : 21-Mar-2006 14:01

Page: 1

Sample: DHR-10

Note : - in PEG standard

Inlet : Reserv.

$\mathrm{RT}: 0.30 \mathrm{~min}$

Elements : C 60/0, H 90/0, O 6/0, N $3 / 0$

Ion Mode : FAB+

Mass Tolerance : $3 \mathrm{mmu}$

Scan\# : $(1,5)$

Unsaturation (U.S.) : $-0.5-10.0$

U.S. Composition<smiles>CNC(=O)[C@H](N)Cc1ccccc1</smiles>

11

Chemical Formula: $\mathrm{C}_{10} \mathrm{H}_{14} \mathrm{~N}_{2} \mathrm{O}$

Exact Mass: 178.11

[ Theoretical Ion Distribution ]

Molecular Formula : C10 H15 N2 O

(m/z 179.1184, MW 179.2419, U.S. 4.5)

Base Peak : 179.1184, Averaged MW : 179.2428(a), $179.2435(\mathrm{w})$

$\mathrm{m} / \mathrm{z} \quad \mathrm{INT}$.

179.1184100 .0000

$180.1215 \quad 12.1202$

181.12410 .8717

$182.1265-0.0467$

$183.1291 \quad 0.0018$ 


\section{HRMS for 12}

[ Elemental Composition ]

Data : J6.617hr-001

Sample: DHR-11

Note : - in PEG standard

Inlet : Reserv.

$\mathrm{RT}: 0.34 \mathrm{~min}$
Elements : C $60 / 0, \mathrm{H} 90 / 0, \mathrm{O} 6 / 0, \mathrm{~N} 3 / 0$

Date : 21-Mar-2006 13:51

Page: 1

Mass Tolerance: $3 \mathrm{mmu}$

Unsaturation (U.S.) : $-0.5-10.0$

Ion Mode : FAB+

Scan\# : $(1,6)$

Observed $\mathrm{m} / \mathrm{z}$ Int $\%$ Err [ppm / mmu]

U.S. Composition

$\begin{array}{llllllllllll}403.2570 & 100.0 & -6.5 / & -2.6 & 7.5 & \text { C } 23 & \text { H } & 35 & 0 & 4 & \text { N } 2\end{array}$

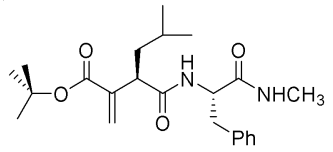

12

Chemical Formula: $\mathrm{C}_{23} \mathrm{H}_{34} \mathrm{~N}_{2} \mathrm{O}_{4}$ Exact Mass: 402.25

[ Theoretical Ion Distribution ]

Molecular Formula : $223 \mathrm{H} 35 \mathrm{~N} 204$

(m/z 403.2597, MW 403.5419, U.S. 7.5)

Base Peak : 403.2597, Averaged MW : $403.5437(\mathrm{a}), \quad 403.5444(\mathrm{w})$

$\mathrm{m} / \mathrm{z} \quad \mathrm{INT}$.

403.2597100 .0000

404.262926 .9935

$405.2658 \quad 4.3015$

$406.2685 \quad 0.5062$

$407.2711 \quad 0.0477$

$408.2737 \quad 0.0038$

$409.2763 \quad 0.0003$

\section{HRMS for 13}

[ Elemental Composition ]

Data : J6.616hr-002

Sample: DHR-12

Note : - in PEG standard

Inlet : Reserv.

$\mathrm{RT}: 0.38 \mathrm{~min}$

Elements : C $60 / 0, \mathrm{H} 90 / 0, \mathrm{O} 6 / 0, \mathrm{~N} 4 / 0$

Date : 21-Mar-2006 13:48

Page: 1

Mass Tolerance : $3 \mathrm{mmu}$

Unsaturation (U.S.) : $-0.5-10.0$

Ion Mode : FAB+

Scan\# : $(1,6)$

$\begin{array}{clrrrr}\text { Observed m/z } & \text { Int\% } & \text { Err }[\mathrm{ppm} / \mathrm{mmu}] & \text { U.S. } & \text { Composition } \\ 347.1990 & 100.0 & +5.4 / 4+1.9 & 7.5 & \text { C } 19 \text { H } 27 \text { O } 42\end{array}$

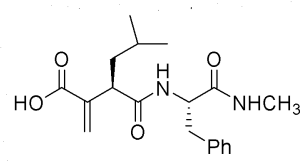

13

Chemical Formula: $\mathrm{C}_{19} \mathrm{H}_{26} \mathrm{~N}_{2} \mathrm{O}_{4}$ Exact Mass: 346.19

[ Theoretical Ion Distribution ]

Molecular Formula : C19 H27 N2 O4

$(\mathrm{m} / \mathrm{z} \quad 347.1971, \mathrm{MW} \quad 347.4344, \mathrm{U} . \mathrm{S} . \quad 7.5)$

Base Peak : 347.1971 , Averaged MW : 347.4357 (a), $347.4364(\mathrm{w})$

$\mathrm{m} / \mathrm{z} \quad$ INT.

347.1971100 .0000

$348.2003 \quad 22.4246$

349.20293 .1973

$350.2056 \quad 0.3417$

$351.2081 \quad 0.0294$

$352.2107 \quad 0.0021$

$353.2132 \quad 0.0001$ 


\section{HRMS for 14}

[ Elemental Composition]

Data : J6.615hr-001

Sample: DHR-14

Note : - in PEG standard

Inlet : Reserv.

$\mathrm{RT}: 0.20 \mathrm{~min}$

Elements : C 60/0, H 90/0, O 6/0, N 4/0, S $1 / 0$

Mass Tolerance : $3 \mathrm{mmu}$

Unsaturation (U.S.) : $-0.5-10.0$

Date : 21-Mar-2006 13:41

Page: 1

Observed $\mathrm{m} / \mathrm{z}$ Int\% Err [ppm / mmu] U.S. Composition

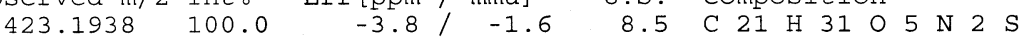

[ Theoretical Ion Distribution ]

Molecular Formula : C21 H31 N2 O5 S

$(\mathrm{m} / \mathrm{z}$ 423.1954, MW 423.5535, U.S. 8.5)

Base Peak : 423.1954, Averaged MW : $423.5534(\mathrm{a}), \quad 423.5545(\mathrm{w})$

$\mathrm{m} / \mathrm{z}$ INT.

423.1954100 .0000

424.198525 .5364

$425.1960 \quad 8.5592 * * * * *$

426.19731 .5962 *

$427.1983 \quad 0.2442$

$428.1998 \quad 0.0300$

$429.2015 \quad 0.0031$

$430.2033 \quad 0.0003$

\section{HRMS for 16}

[ Elemental Composition ]

Data : J6.612hr-001

Sample: DHR-15/2

Note : - in PEG standard

Inlet : Reserv.

$\mathrm{RT}: 0.20 \mathrm{~min}$

Elements : C 60/0, H 90/0, O 5/0, N $4 / 0$
Mass Tolerance

$\begin{array}{ll}\text { Mass Tolerance } & : 3 \mathrm{mmu} \\ \text { Unsaturation (U.S.) } & :-0.5-10.0\end{array}$

$\begin{array}{clr}\text { Observed m/z } & \text { Int\% } & \operatorname{Err}[\mathrm{ppm} / \mathrm{mmu}] \\ 362.2101 & 100.0 & +2.1 / \mathrm{+}\end{array}$
Date : 21-Mar-2006 13:32

Ion Mode : FAB+

Scan\#: $(1,4)$

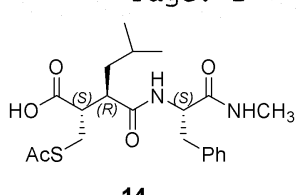

14

Chemical Formula: $\mathrm{C}_{21} \mathrm{H}_{30} \mathrm{~N}_{2} \mathrm{O}_{5} \mathrm{~S}$ Exact Mass: 422.19 


\section{HRMS for 17}

[ Elemental Composition ]

Data : J6.614hr-001

Sample: DHR-14

Note : - in PEG standard

Inlet : Reserv.

$\mathrm{RT}: 0.19 \mathrm{~min}$

Elements : C $60 / 0, \mathrm{H} 90 / 0$

Mass Tolerance : $3 \mathrm{mmu}$

Unsaturation (U.S.) : $-0.5-10.0$

$\begin{array}{clr}\text { Observed } \mathrm{m} / \mathrm{z} & \text { Int\% } & \text { Err }[\mathrm{ppm} / \mathrm{mmu}] \\ 438.2083 & 100.0 & +1.5 / \mathrm{t} / 0.7\end{array}$ $+4.6 /+2.0$

$8.0 \quad \mathrm{C} 23 \mathrm{H} 3406 \mathrm{~S}$

$8.5 \mathrm{C} 21 \mathrm{H} 3205 \mathrm{~N} 3 \mathrm{~S}$
U.S. Composition

Page: 1

Ion Mode : FAB+

Scan\# : $(1,3)$

$4 / 0$, S $1 / 0$

$\mathrm{HO}-\mathrm{N}$

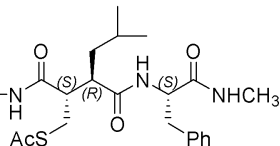

17

Chemical Formula: $\mathrm{C}_{21} \mathrm{H}_{31} \mathrm{~N}_{3} \mathrm{O}_{5} \mathrm{~S}$ Exact Mass: 437.2

[ Theoretical Ion Distribution ]

Molecular Formula : C21 H32 N3 $05 \mathrm{~S}$

$(\mathrm{m} / \mathrm{z} \quad 438.2063, \mathrm{MW} \quad 438.5682$, U.S. 8.5)

Base Peak : 438.2063, Averaged MW : $438.5681(\mathrm{a}), \quad 438.5691(\mathrm{w})$

$\mathrm{m} / \mathrm{z}$ INT.

438.2063100 .0000

439.209325 .9188

440.20698 .6568

$441.2081 \quad 1.6290$

442.20910 .2503

$443.2106 \quad 0.0310$

$444.2122 \quad 0.0032$

$445.2140 \quad 0.0003$ 